\title{
SETTING UP A GRID SIMULATOR
}

\author{
A. Notholt ${ }^{1}$, D. Coll-Mayor ${ }^{2}$, A. Engler ${ }^{1}$
}

${ }^{1}$ Institut für Solare Energieversorgungstechnik (ISET).

Königstor 59. D-34119 Kassel

anotholt@iset.uni-kassel.de

${ }^{2}$ Department of Physics. University of Balearic Islands

Ctra. Valldemossa, km 7.5 / E-07122 Balearic Islands

\begin{abstract}
The test and validation of prototypes in the area of Distributed Generation is often related to the effect of these devices on the electrical grid. However, due to the scale of the mentioned equipment it is difficult to observe this effect when the device interacts with the main grid. A first approximation to this behavior can be simulated using a model which interacts with a simulated environment, with a software tool. When the software model is well defined, the prototyping phase starts and new testing will be required. The purpose of this work is to develop a test stand for such devices which would mimic some of the most important parameters of a grid. The impact of the tested device in the grid can therefore be adjusted. This grid simulator will be used for stability studies in the introduction of Distributed Generation Devices as well as Renewable Energy Sources in the grid and it can be seen as a scaled model of the real grid. This work was implemented in the DeMoTeC laboratory in the Institut für Solare Energieversorgungstechnik (ISET) in Kassel (Germany) and it has been used for testing minigrids connected with the main grid.
\end{abstract}

Keywords: Distributed generation; Power Grid Simulator; Renewable Energy Sources Integration; Energy infrastructures.

\section{Introduction}

Simulation tools bring, in general, a considerable reduction in development times of consumer products as well as test phases. In the case of power systems, they permit to visualize complex cases such as stability problems, power flows or electromagnetical transients in a fast, relatively inexpensive way.

Examples of such tools are the Power System Simulator for Engineering PSS/E [1] and the Alternative Transients Program ATP-EMTP [2], These tools are widely used in stability studies of power transmission and distribution grids among others. Although initially thought for largerscale power systems, these tools have been also applied in studies in the areas of Distributed Generation and Demand Side Management with successful results [3].

However, despite the clear advantages of pure Computer-Assisted simulations, there is the necessity of, at some point, conduct tests of prototypes under more real conditions. These experiments normally require interacting with the grid to estimate their effect into the power system. The impact of the prototype in the local energy grid is most of the times regarded as insignificant. Therefore the need for having a test stand in which new configurations, equipment or control techniques in the area of Distributed Generation could be tested.

The work presented proposes a simple, effective way of setting up and configuring a Grid Simulator for its use as a test stand of Distributed Generation (DG) and Demand Side Management (DSM) techniques, using existing hardware of the DeMoTeC laboratory in Germany.

\section{Main Requirements}

In order to build up a laboratory simulator for analyzing the impact of DG and DMS in the power grid, some requirements were identified. First, the Simulator had to be able to represent a tiny to small grid (Peak Power in the range of $1 \mathrm{GW}$ ) and be scalable. The parameters of this simulated grid had to be easily changeable. Since the primer objective of the Simulator was to be use for analyzing the effect of DG and DMS, it had to be affectable by small loads. The last requirement was to use commercial hardware.

\section{Hardware description}

The hardware used comprises a $80 \mathrm{~kW}$ synchronous machine with automatic voltage compensation (field control) mechanically coupled to a shunt-connected DC machine. The DC link current is electronically controlled and the terminals of the synchronous machine are connected to an interconnection board where the equipment will be tested.

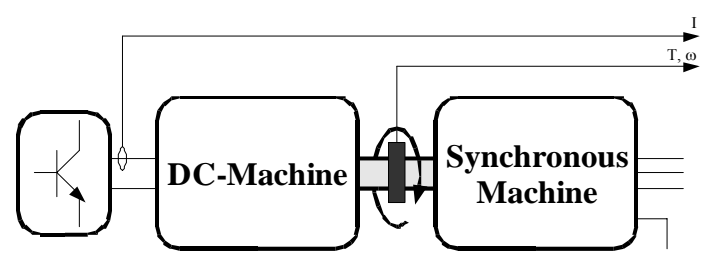

Figure 1: Current Hardware

Additionally, a torque as well as a speed and current sensors are installed. The block diagram of the equipment used is shown in Figure 1.

The original control of the DC machine is done through a Matlab ${ }^{\circledR}$ interface which is depicted in figure 2. The frequency is set to a fixed value of usually $50 \mathrm{~Hz}$. When the torque demanded by the synchronous generator 
grows up, the DC machine rapidly increases the power output in order to compensate the demanded power and maintain the speed constant. An additional torque limiter is implemented for security. In this control the effect of the control curves of different generators and other components in the simulated network are not contemplated.

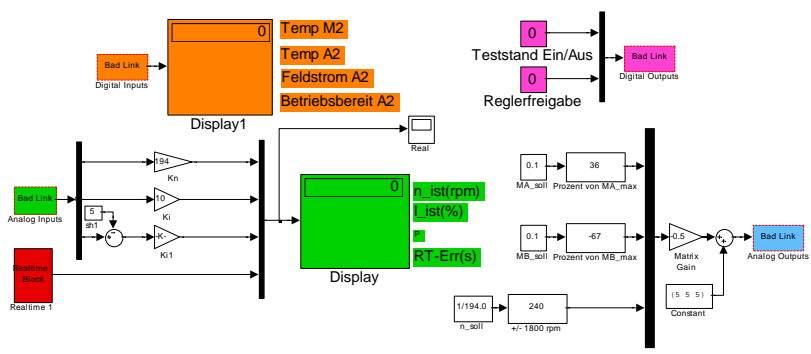

Figure 2: Matlab Control tool of the DC generator

\section{General Principles}

The control technique proposed in this paper consists in incorporating the frequency response component into the current control; this is, by measuring the output power and the current speed, the system should be able to generate the suitable speed setpoint that would represent the theoretical grid frequency.

Since the deviations of power and frequency relevant for the test cases are relatively small, a linearized model (small disturbance model) of a power grid will be used.

\section{A. Grid Model}

In order to provide a simple, but standard test case, an IEEE standard test case has been used. This Test Case represents a portion of the American Electric Power System (in the Midwestern US) as of February, 1962 [4], see figure 3 . This 14 nodes test case provides enough nodes to represent a small isolated power system, while keeping the model simple.

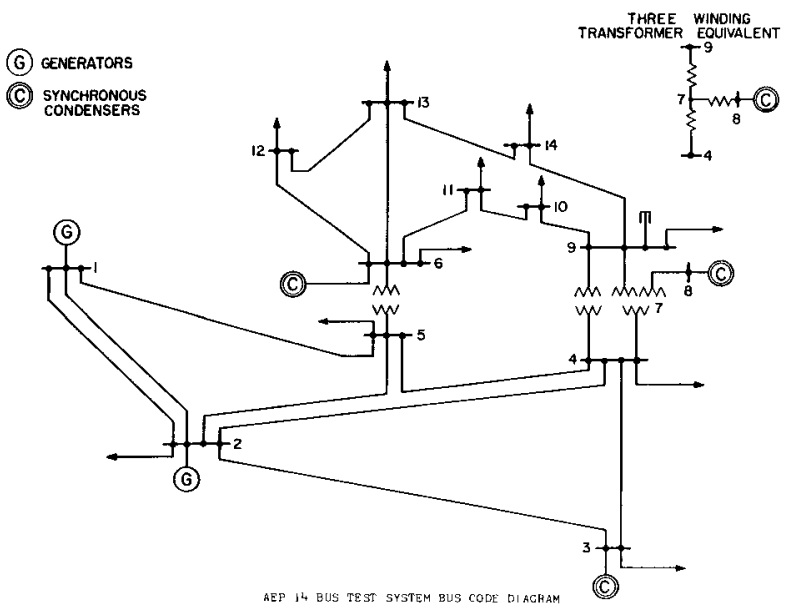

Figure 3: Simulation of the IEEE 14 Nodes Test Case
However, this test case is used for static power flow calculations and therefore lacks of the dynamical parameters of the generators. These parameters can be found in several studies and references such as [5].

\section{B. Generators}

In the proposed grid, five different generation facilities were considered, three standard thermal power stations and two combined cycles. Figure 4, represents the general form of the model used to simulate the different generation plants. This comprises: The primary control of the plant; And the general transfer function of the aggregated plant and involved controls.

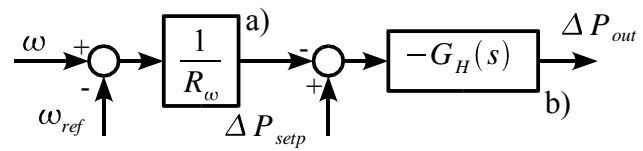

Figure 4: General linearized model for a generation plant

The frequency -or primary- control has been adjusted adequately to each generator size based on the power flows reported in the IEEE Test Case. The transfer function $\mathrm{G}_{\mathrm{H}}$, depends on the type of generator; for this case, typical values obtained from have been used and the inertia values of the generator machines have been based on reference [6].

The representation of the control with the five generators is given in figure 5 .

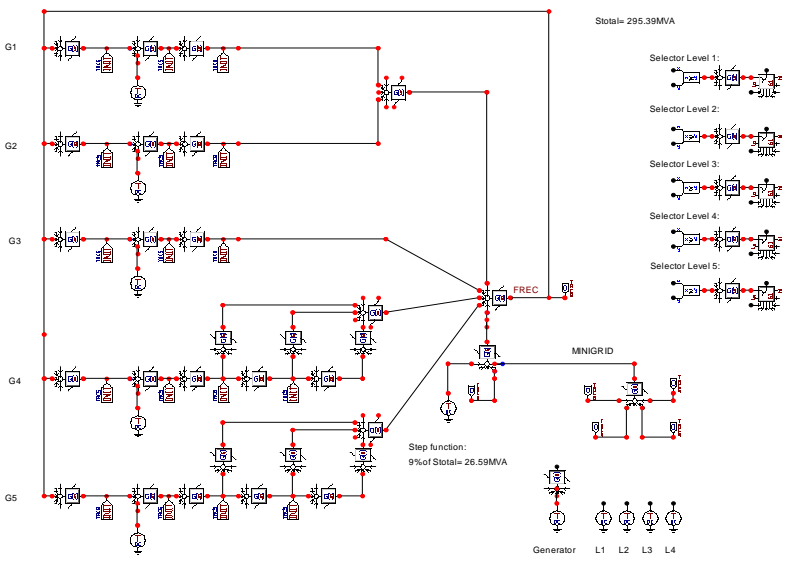

Figure 5: Model of the control of the minigrid coupled to the IEEE 14 Node Test

The limitations of the primary control are an important characteristic of the implemented model. These limitations are related to the fact that at any given time slot (of usually 15 minutes) the primary control is able to vary the generator's output in the range of $+/-5 \%$.

\section{Loads and Lines}

The consuming components of the system such as lines and loads have a response to sudden unbalances between the generated and the consumed power of the type: 


$$
\frac{\Delta f}{\Delta P_{\text {syst }}}=\frac{K_{s}}{1+s T_{s}}
$$

$n$ equation (1), $K_{S}$ and $T_{S}$ are defined as the delay of the system response to a power unbalance between generation and production. Knowing that $\mathrm{K}_{\mathrm{S}}=1 / \mathrm{D}$, D is defined as the response -in terms of consumed power- of the system to a change in the system frequency. Considering all linear models for lines and loads, the resulting constant $\mathrm{D}$ was found to be $0.03 \mathrm{puMW} / \mathrm{Hz}$, power base of 100MVA. This value of the D parameter was accepted since the value of $\mathrm{D}$ for a $1 \mathrm{GW}$ grid would be around $0.02 \mathrm{puMW} / \mathrm{Hz}$ and for a $5 \mathrm{GW}$ would be $0.01 \mathrm{puMW} / \mathrm{Hz}[7]$.

\section{Control Implementation}

As justified above, the control's task is to give a speed setpoint to the machine, which would represent the current power consumption on the demand load. Since a small disturbances model is being used, a steady state must be reached before the experiment can run and the simulated model can be used. Once this state is reached, the values of power and frequency are set up as constants and have the function of converting the actual values of power and frequency into deviations from the steady state. Figure 6 illustrates this conversion.

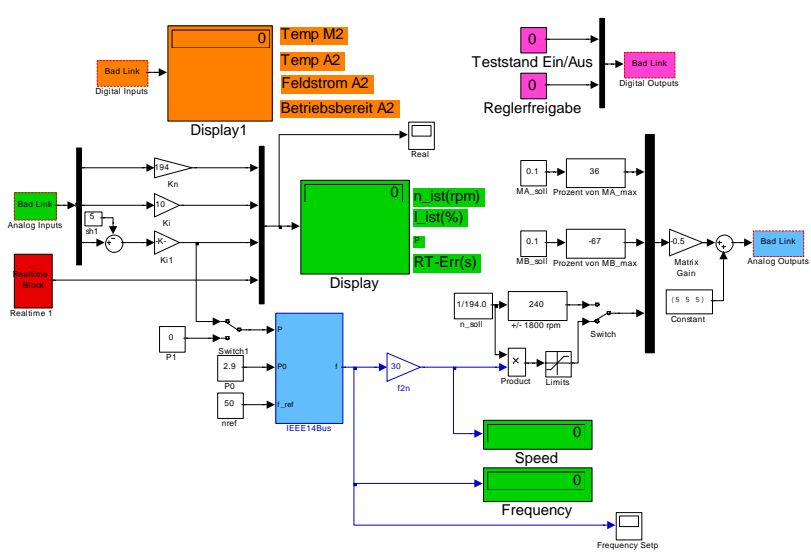

Figure 6: Matlab Control block of the grid simulator

An analogue conversion from the frequency should be done in order to provide the speed setpoint to the further control algorithm. The control block is therefore compounded by 3 inputs: Actual demanded power, steady state speed and steady state power consumption and gives the setpoint frequency as output. The block called IEEE 14bus in figure 7 represents the grid behaviour. The grid model is developed in a per-unit system, allowing an easy scaling of the model to suit different experiment configurations.

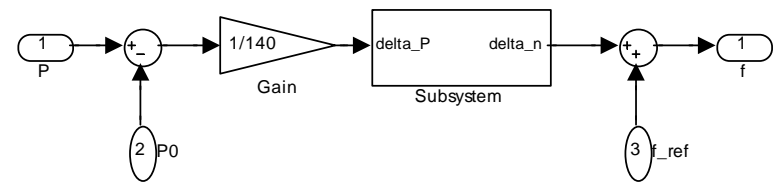

Figure 7: IEEE 14bus block
Once the data is converted to the required format, the grid is simulated with the block called subsystem. This block contains the above mentioned equations which are representing the grid, see figure 8 .

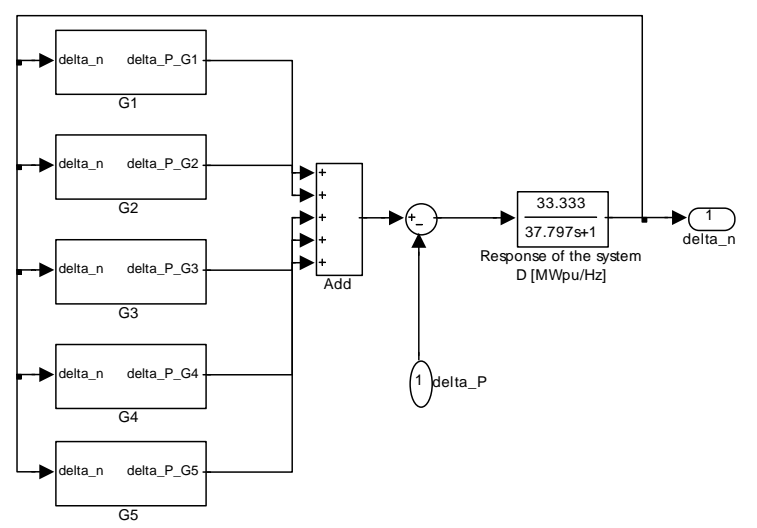

Figure 8: IEEE 14bus block subsystem

The grid is built up with 5 generators, the different generators have specific characteristics, which can be seen opening the respective blocks. The power response of the grid for a varying frequency (D) is given as transfer function.

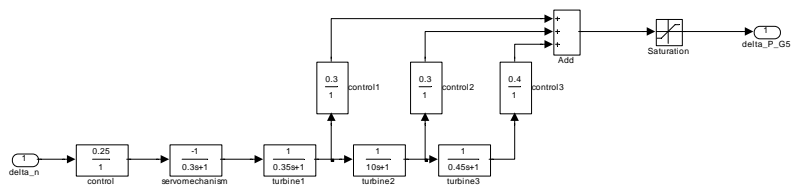

Figure 9: Generator 5

As an example, the generator 5 (G5) is depicted in figure 9. This generator is a combined cycle plant with three turbines of high and low pressure.

\section{Example of Experiment performed with the Grid Simulator}

The experiment performed consisted in testing the response of the grid in front of a power input which destabilizes the system [8], and conduct the grid frequency out of the margins accepted by the system.

This experiment was simulated using a standard software tool and comparing the result with the result obtained by using the simulator described in this paper.

\section{A. Software simulation}

The simulation was done by using a software tool. In the described power grid a step of power is simulated; this step is enough for, knowing the characteristics of the generators and the constant $\mathrm{D}$ of the system, to drive the whole system out of the frequency acceptable margin.

Figure 10 represents the response of the grid using a software tool in front of a 0.26puMVA step function. This response of the main grid is a decrease in the 
frequency that achieves a new equilibrium point at $49.45 \mathrm{~Hz}$, this new equilibrium is out of acceptable range.

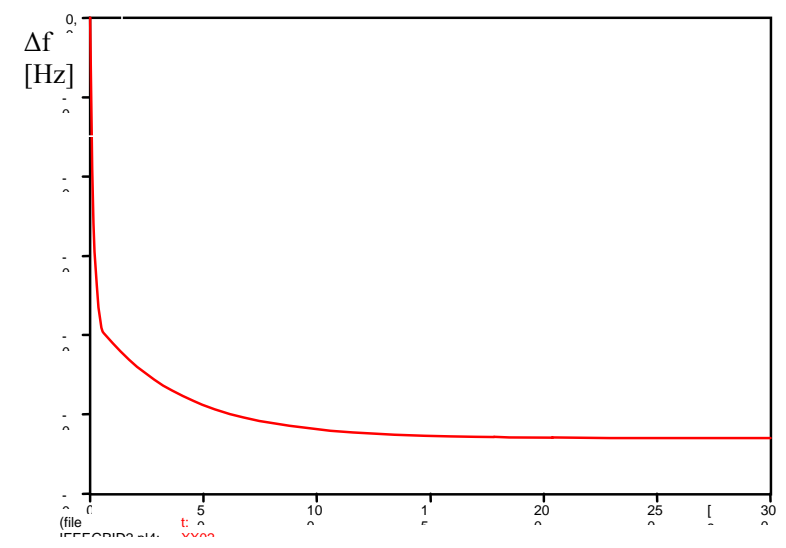

Figure 10: Response of the grid to a 0.26 puMVA step

Adding load shedding response for the system, after a while the frequency starts to recover, see on figure 11.

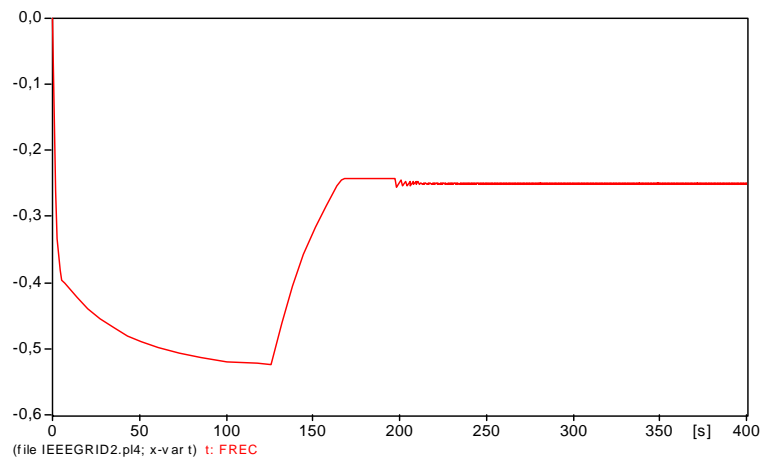

Figure 11: Frequency Response of the system to a 0.26 puMVA and load shedding

The instabilities that can be seen in figure 11 are provoked by the reconnection of the lines when the frequency set point is achieved. In the real model this instability has been corrected by using a time delay in the reconnection process.

\section{B. Grid Simulator}

Representing the same situation with the grid simulator using the standard control, before the modifications proposed in this paper, the result of the frequency behavior of the power grid is shown in figure 12. The matlab control tool used for controlling the DC motor keeps the frequency almost constant at $50 \mathrm{~Hz}$ for small disturbances.

If the modified control for the grid simulator is used the result is a variation in the frequency of the grid, this variation can be seen in figure 13 .

Adding also the different lines of loads in the load shedding schema the result of the grid simulator behavior is given in figure 13 .

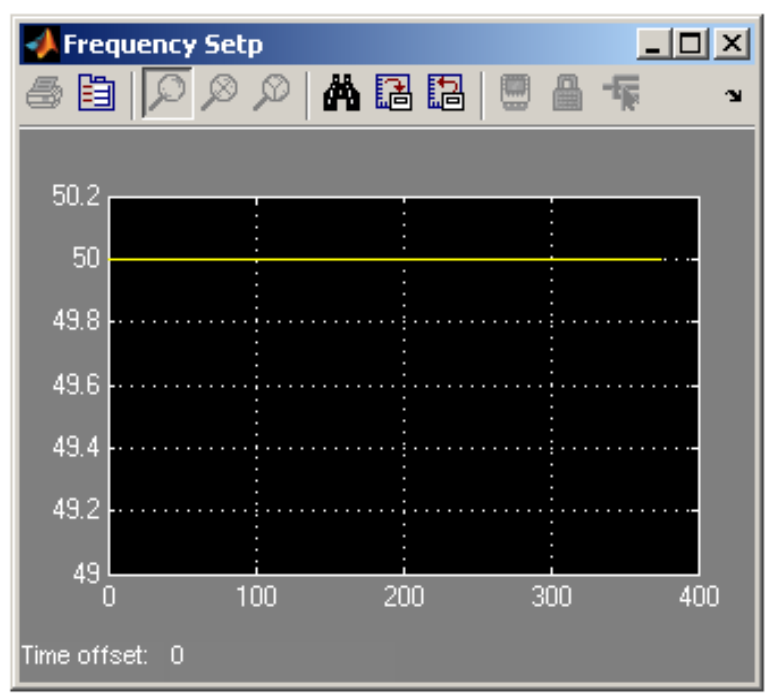

Figure 12: Output of the frequency in the grid while using the DC motor control before modifications

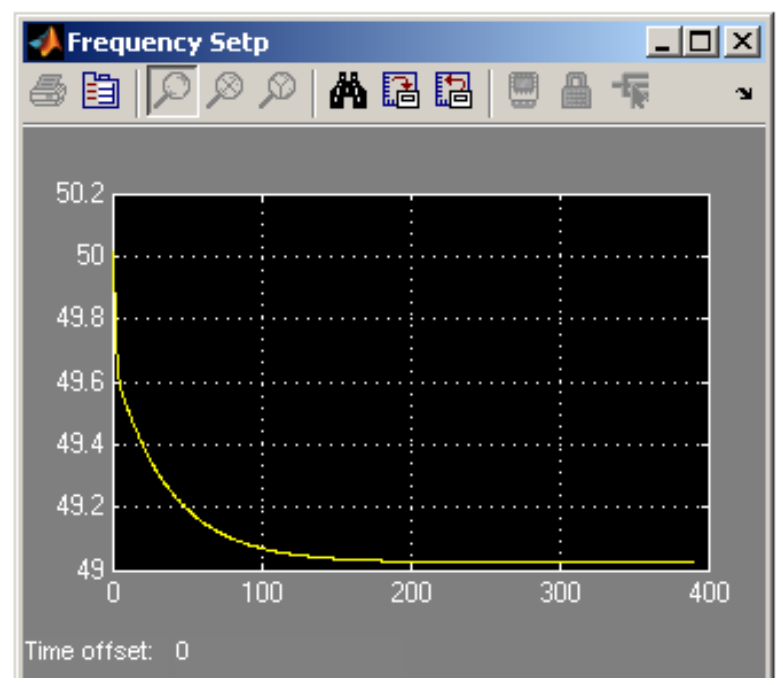

Figure 12: Output of the frequency in the grid while using the proposed DC motor control

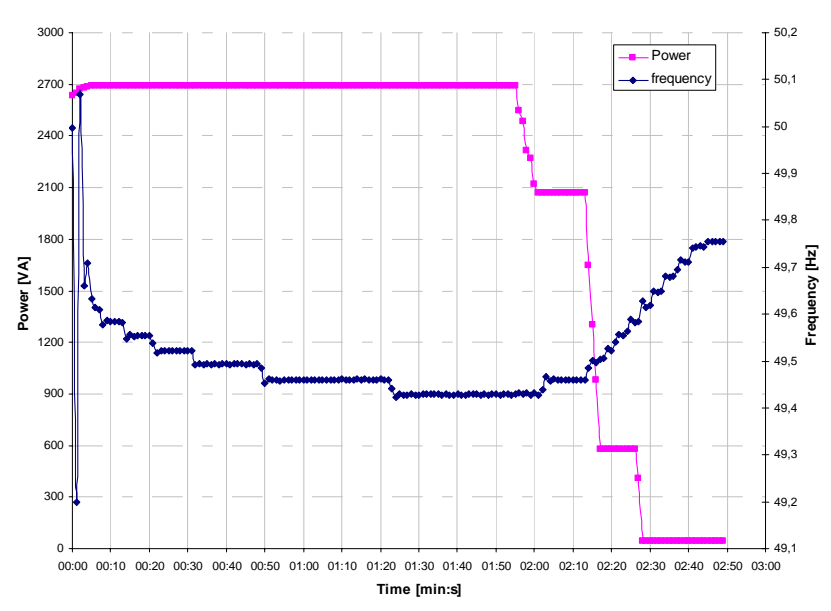

Figure 13: Frequency recovering in the grid while using the proposed DC motor control and 3-lines-load shedding technologies 
The reaction of the grid simulator represents the real reaction of a small power grid subjected to small disturbances. This reaction is consistent with the one simulated by using software tools.

\section{Conclusions}

The grid simulator has been used in representing the behavior of the power grid when introducing DG and RES units. The grid simulated is a scaled model of the real grid and can be easily adapted for representing different kinds of grids and at different scales. This ability makes of it a useful tool in order to estimate the effect of large integration of DG devices in different kind of power grids, as well as the analysis of Demand Side Management techniques when disconnecting loads by underfrequency.

\section{References}

[1] PSS/E $\mathrm{E}^{\mathrm{TM}}$ version 30. Comprehensive analysis software for use in transmission system studies. Siemens, 2005.

[2] Information downloable from: http://www.emtp.org/

[3] Strauss, P. and Engler, A. AC coupled PV hybrid systems and microgrids-state of the art and future trends. Proceedings of 3rd World Conference on Photovoltaic Energy Conversion. Volume 3, 12-16 May 2003 Page(s):2129 2134. Osaka 2003. Japan.

[4] IEEE Working Group on a Common Format for the Exchange of Solved Load Flow Data. Common Data Format for the Exchange of Solved Load Flow Data. IEEE Transactions on Power Apparatus and Systems, Vol. PAS92, No. 6, November/December 1973, pp. 1916-1925.

[5] P. M. Anderson and A. A. Fouad, Power System Control and Stability. ISBN: 0-471-23862-7. Willey- IEEE Press.

[6] Kodsi, S.K.M. and Claudio A. Cañizares, C.A. Modeling and simulation of IEEE 14 Bus System with FACS Controllers. Technical Report \#2003-3. University of Waterloo, Waterloo March 2003.

[7] Gómez Expósito, A., Análisis y operación de sistemas de energía eléctrica. Ed. McGrawHill. ISBN 94-481-3592-X. Madrid 2002.

[8] D. Coll-Mayor. PhD thesis: The minigrid as a Grid Supporting element. Kassel, 2005. In Review process. 